\title{
Microstrip line modelling in the presence of interfering metal strips according to their orientation.
}

\author{
Thierry Le Gouguec, Pierre-Marie Martin \\ Université de Brest ; CNRS, UMR 6285 Lab-STICC, \\ 6 avenue Victor Le Gorgeu, CS 93837, 29238 Brest cedex 3, France \\ Thierry.legouguec@univ-brest.fr ; Pierre-Marie.Martin@univ-brest.fr
}

\begin{abstract}
In the context of PCB technology, this paper deals with the measurement and simulation of propagation from a microstrip line disturbed by a metal ribbon placed just above it. We investigate the influence of the angular orientation of the interfering strip and we highlight the presence of disturbances on transmission S-parameters. Lastly, we propose a model of these ribbon orientation effects, which is validated by the experimental results.
\end{abstract}

\section{Introduction}

Higher integration densities and an increase in the number of metal layers in PCB structures as well as the use of new concepts such as "System in Package" (SiP) or "System on Package” (SoP) [1], all cause a significant rise in the possible interactions between various interconnects or between interconnects and power/ground grids. Furthermore, an increase in clock frequencies makes these interactions more problematic for signal integrity.

Effects induced by perpendicular metal grids on the characteristics of transmission lines or coupled lines in a multilayered architecture were highlighted in [2] and [3].

In this communication, our investigations aim at modeling the impact by metal disturbances whatever their orientation on signal propagation in microstrip line. The context of this study is multilayered PCB circuits.

\section{Disturbing effect of one parasitic strip}

Fig. 1 shows a photograph of the measurement structure under consideration. This device consists of a main $50 \Omega$ microstrip copper line measuring length $L=60 \mathrm{~mm}$ and width $W=1400 \mu \mathrm{m}$ placed over a FR4-type substrate $\varepsilon_{R}=3.6$ whose thickness is $H_{\text {sub }}=780 \mu \mathrm{m}$ and loss tangent $\tan (\delta)=0.02$. This line is disrupted by a metal ribbon realized on a similar substrate and placed at the upper level. The perturbed line can make an angle with the main line of $\theta=0^{\circ}, 30,60$ or $90^{\circ}$.

This microstrip structure allows us to model the line behaviour in a multilayered PCB environment. We analyzed the impact on transmission by length $\left(L_{P}\right)$ and width $\left(W_{P}\right)$ of the disturbing metal strip and effect of its angle orientation $\theta$ with respect to the line. We also considered floating parasitical ribbons and the case of ground (of main line) connected interfering strips. The S-parameters measurements were made with a HP8720 network analyser over the 0 - 10 $\mathrm{GHz}$ frequency band after a simple SOLT calibration.

Fig. 2 shows both $S_{11}$ and $S_{21}$ (reflection and transmission) coefficients of the MTL line in the presence of a parasitic floating metal strip of length $L_{P}=40 \mathrm{~mm}$, width $W_{P}=1400 \mu \mathrm{m}$ for three $\theta$ angles equal to: $30^{\circ}, 60^{\circ}$ and $90^{\circ}$. The interference line is separated to the MTL line by a substrate $780 \mu \mathrm{m}$ thick. In Fig. 2, we also present the results of the reference MTL alone. About the transmission (Fig2-a), we note the occurrence of several transmission zeroes in the frequency bandpass: 0 - $10 \mathrm{GHz}$, which can be very harmful in signal transmissions [2]. Note that the disturbing line orientation modifies the frequencies at which transmission zeroes occur. For example, we notice a $200 \mathrm{MHz}$ variation of the transmission zero occurrence frequency situated around 4 $\mathrm{GHz}$ when the orientation angle varies between $30^{\circ}$ and $90^{\circ}$.

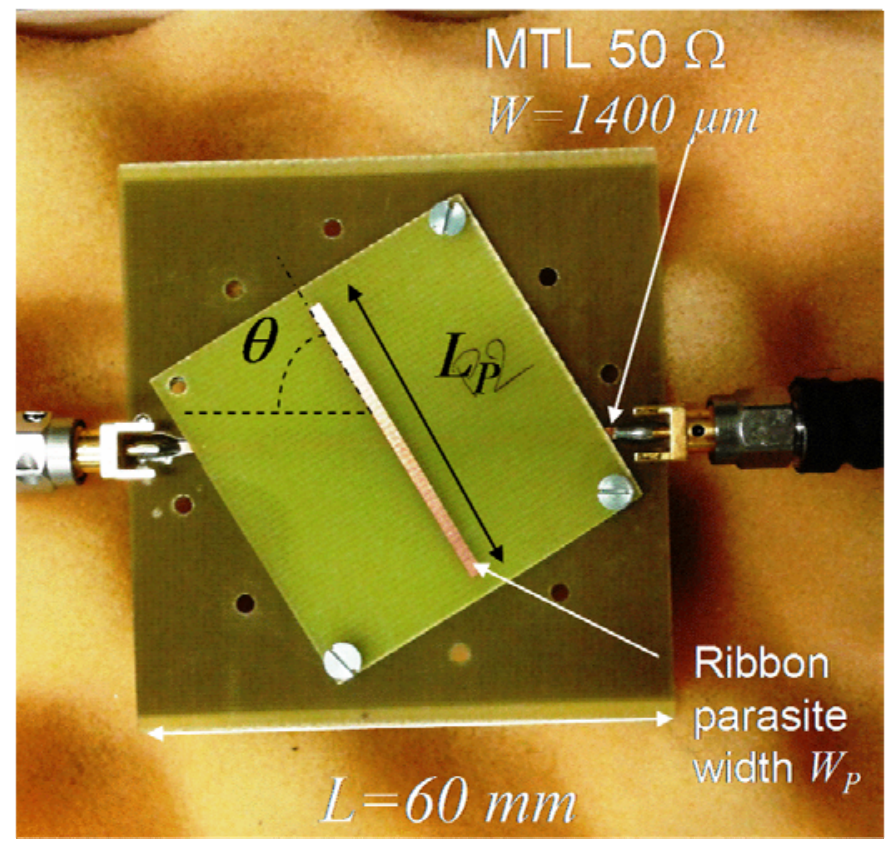

Fig. 1: Top view of the structure under testMicrostrip line (MTL) in the presence of a disturbing floating metal strip.

The $S_{11}$ reflection (Fig. 2-b) doesn't add more useful information than the $S_{21}$ and it is just given here as an indication.

\section{Circuit model}

Based on the previous studies we propose the equivalent circuit model presented in Fig. 3. This model is based on a lumped electric and magnetic coupling between the main microstrip line and the disruptive line. The angle orientation effects result in two inductive $K_{L}$ and capacitive $K_{C}$ coupling coefficients. 

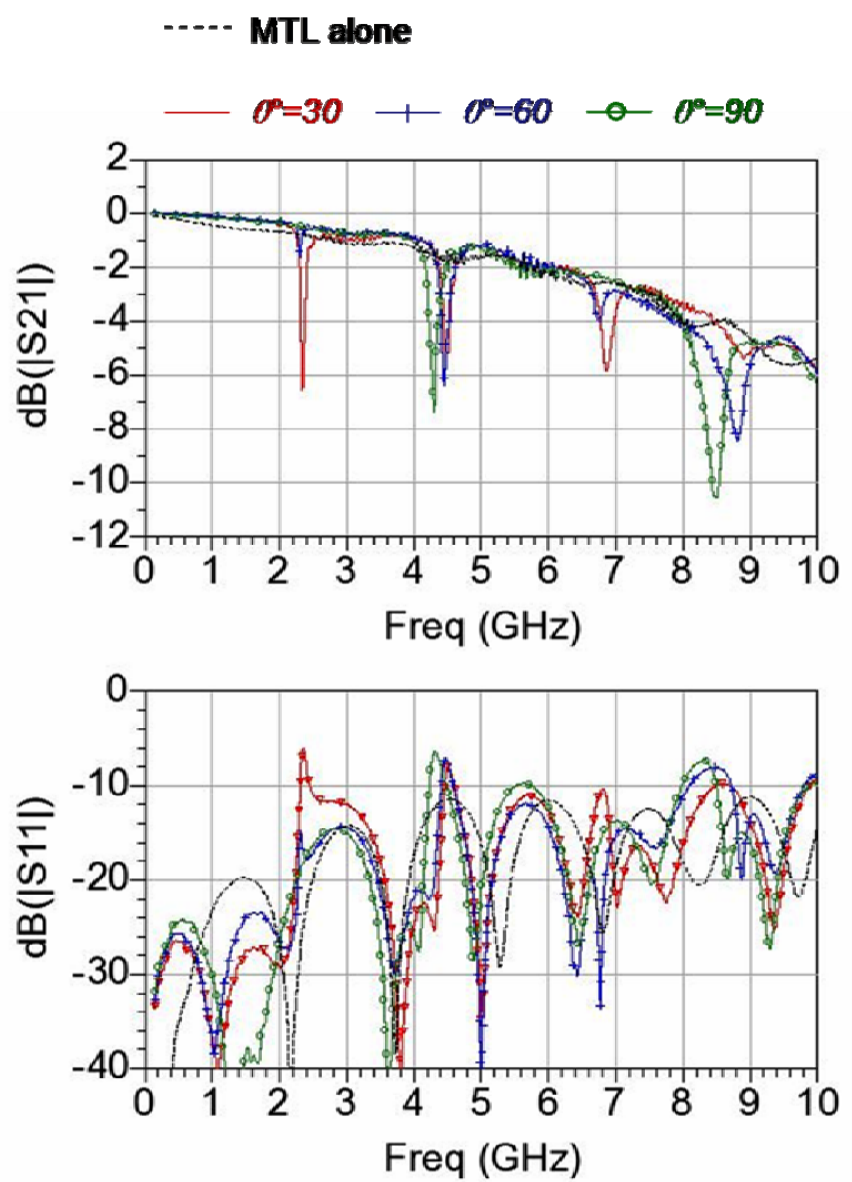

Fig. 2: Measured S parameters for $\theta=30^{\circ}, 60^{\circ}$, and $90^{\circ}$ in case of a floating interfering strip.

These coefficients can be calculated by Q3D extractor software to ANSYS ${ }^{\mathrm{TM}}$ and their evolutions with $\theta$ are presented in Fig. 4. The lumped coupling capacitance $\Gamma$ and the lumped coupling inductance $M$ are related to $K_{C}$ and $K_{L}$ by the following expressions.

$$
\begin{aligned}
& \Gamma=K_{C} \sqrt{C_{1} C_{2}} \\
& M=K_{L} \sqrt{L_{1} L_{2}}
\end{aligned}
$$

Where $C_{1}$ and $C_{2}$ represent the capacitances of the main microstrip line and the interfering ribbon respectively; $L_{1}$ and $L_{2}$ represent the inductances of the MTL and the disturbing conductor in the coupling zone.

The capacitive coupling coefficient $K_{C}$ varies significantly for angles $\theta$ inferior to $45^{\circ}$. Beyond $\theta=45^{\circ}, K_{C}$ coefficient varies only slightly with increasing angle $\theta$. Regarding the inductive coupling coefficient $K_{L}$, we observe a quasi-linear variation. $K_{L}$ value is maximum when the interfering strip is aligned with the line, and of course, $K_{L}$ tends towards zero when the main line and disruptive conductor are perpendicular.
Interfering metal strip

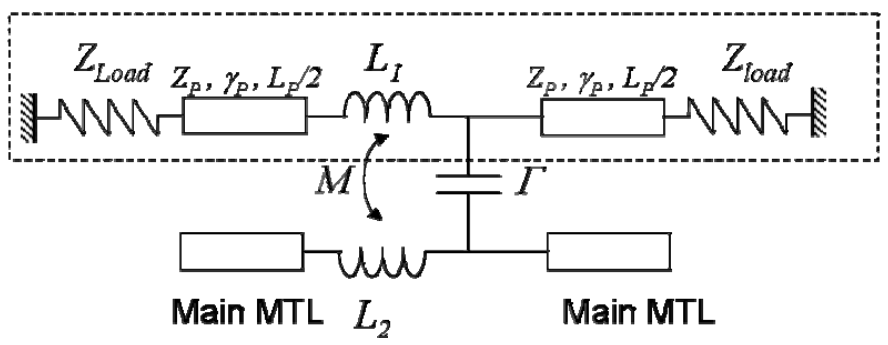

Fig. 3: Equivalent circuit model of a microstrip transmission line (MTL) in presence of an interfering metal strip.

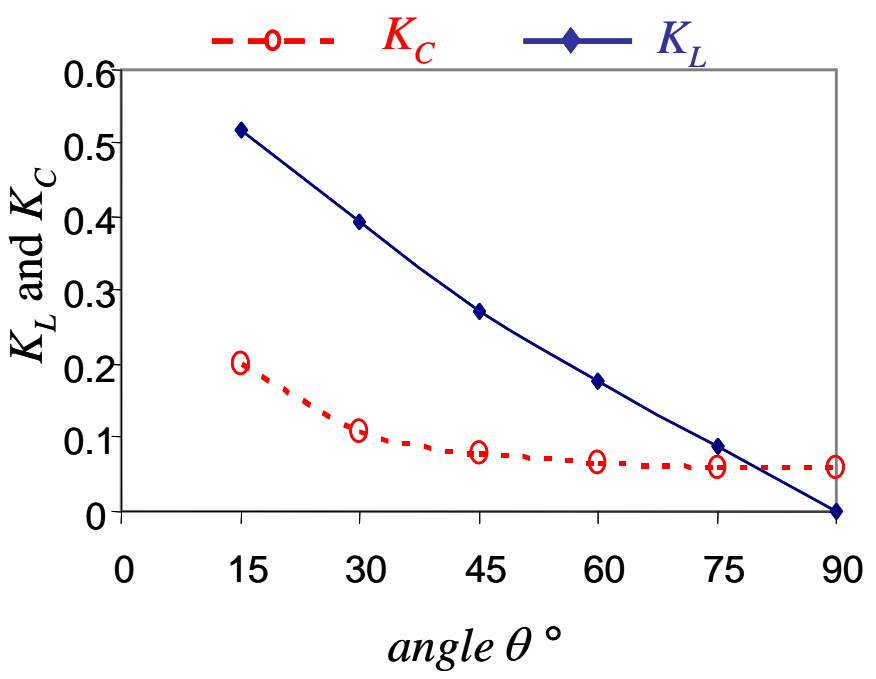

Fig. 4: $K_{C}$ and $K_{L}$ evolution with the orientation angle in the case of $40 \mathrm{~mm}$ long interfering floating strip

In the model, the influence of the length and the width of an interfering strip are mainly taken into account in modelling transmission line of the disturbing conductor. Finally the impedance $Z_{\text {load }}$ can account for the floating character $\left(Z_{\text {load }} \approx\right.$ $\infty)$ or connected to MTL ground $\left(Z_{\text {load }} \approx 0\right)$ of the parasite line.

To validate our method, we compared the simulation and measurement results for several types of disruptive metal strips (different widths $W_{P}$ and lengths $L_{P}$ ) and also for different orientation angle values. For example, Fig. 5 shows comparisons on $S_{21}$ transmission coefficient between the circuit model and the measurements of an interfering floating metal ribbon $L_{P}=40 \mathrm{~mm}$ long, $W_{P}=1400 \mu \mathrm{m}$ wide and for $\theta=60^{\circ}$. There is a good agreement between measurements and circuit modeling simulations. We have also verified our modelling by comparing circuit simulation with HFSS EM results in both cases floating disruptive metal strip and shorted one. These results are presented in Fig.6 and Fig. 7. Here again, EM and circuits modelling results concur closely, which validate our circuit approach. 


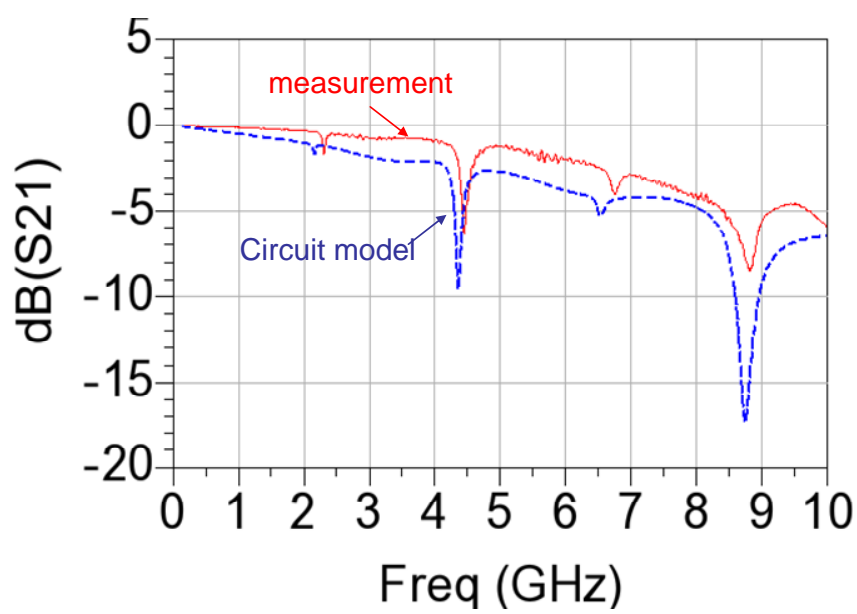

Fig. 5: Measured and modeled $S_{21}$ transmission parameter for $\theta=60^{\circ}$ and $L_{P}=40 \mathrm{~mm}$ (floating disruptive metal strip).

So, this electrical circuit approach can be used to consider and to predict the impact by diverse disturbing metallic lines placed above main lines in multilayered PCB structures.
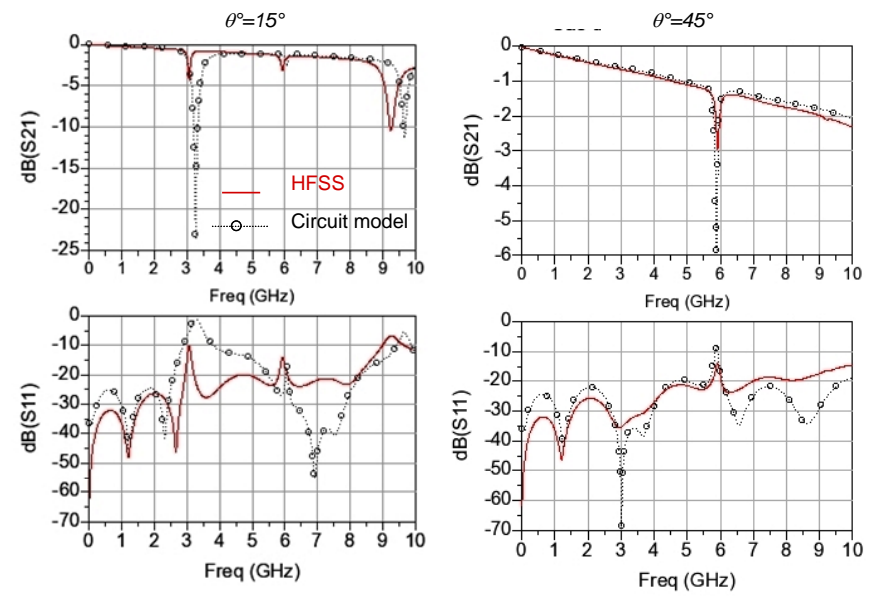

Fig. 6: $S$ parameters of MTL in presence of interfering floating strip forming an angle $\theta=15^{\circ}$ or $\theta=45^{\circ}$.
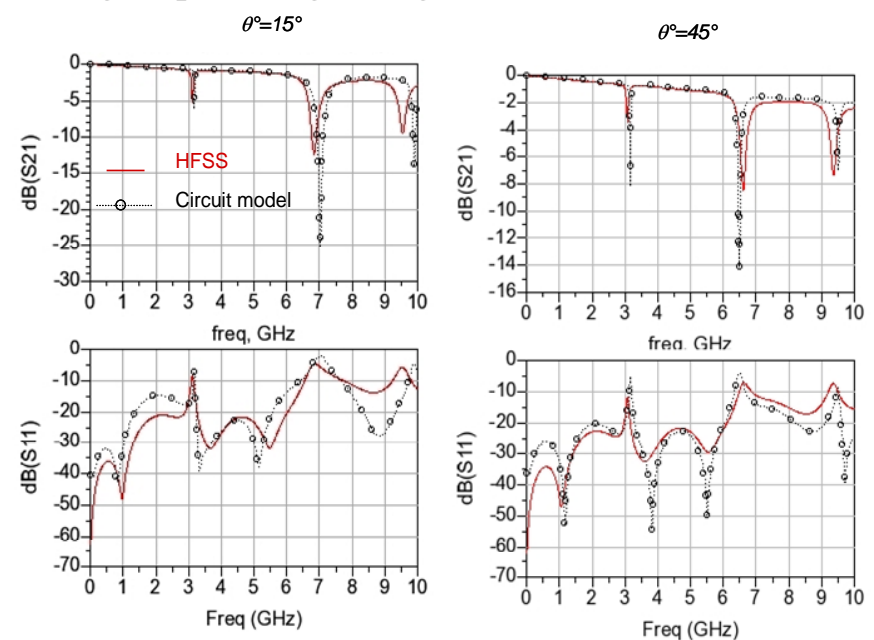

Fig. 7: $S$ parameters of MTL in presence of interfering strip connected to the MTL ground forming an angle $\theta=15^{\circ}$ or $\theta=45^{\circ}$.
The two approaches (EM simulation and circuit modelling) are coherent in both cases, floating parasitic strip or connected to the MTL ground, which validate again our circuit model.

We extend this circuit approach to the case of a microstrip transmission line in presence of metal parasitic grids.

\section{Circuit model of MTL in presence of an arbitrarily oriented metal grid.}

Fig. 8 presents an example of the structures considered in this section. We consider a $40 \mathrm{~mm}$ long metal grid composed of 10 parallel strips. These grids can be oriented in relation with the microstrip line. We also have considered the cases of a floating grid and connected to the MTL ground. This study was realized using both EM simulations and measurements.

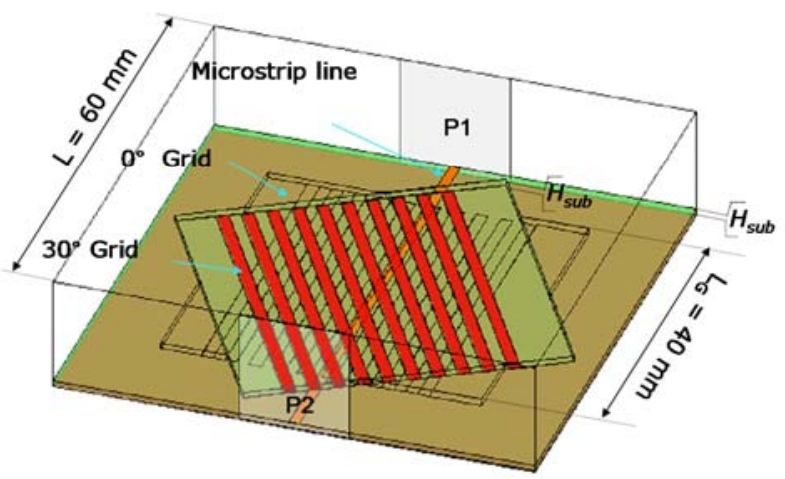

Fig.8: Microstrip line in presence of a 10 strip metal grid.

To model these structures by a circuit, we have considered each strip as independent. The grid can be modelled by a succession of elementary cells such as those presented in the previous section. Fig. 9 presents the proposed equivalent circuit model of a microstrip line disrupted by a 10 strip metal grid.

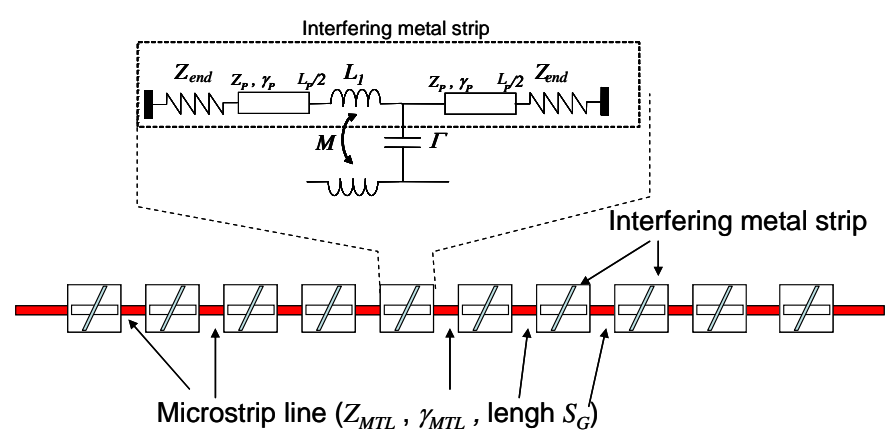

$S_{G}=$ space between 2 grid strips

Fig. 9: Circuit model of a MTL in presence of a 10 strip metal grid.

To validate this circuit approach, we have considered a 10 strip metal grid $W_{G}=1.4 \mathrm{~mm}$ wide, $S_{G}=1.4 \mathrm{~mm}$ spaced and graved on FR4 substrate (thickness $H_{\text {sub }}=780 \mu \mathrm{m}$, relativity permittivity $\varepsilon_{R}=3.6$ and loss tangent $\left.\tan (\delta)=0.02\right)$. The floating grid is $L_{G}=40 \mathrm{~mm}$ long and can be oriented of $0^{\circ}, 30^{\circ}$, $60^{\circ}$ or $90^{\circ}$ in relation with the microstrip line. 


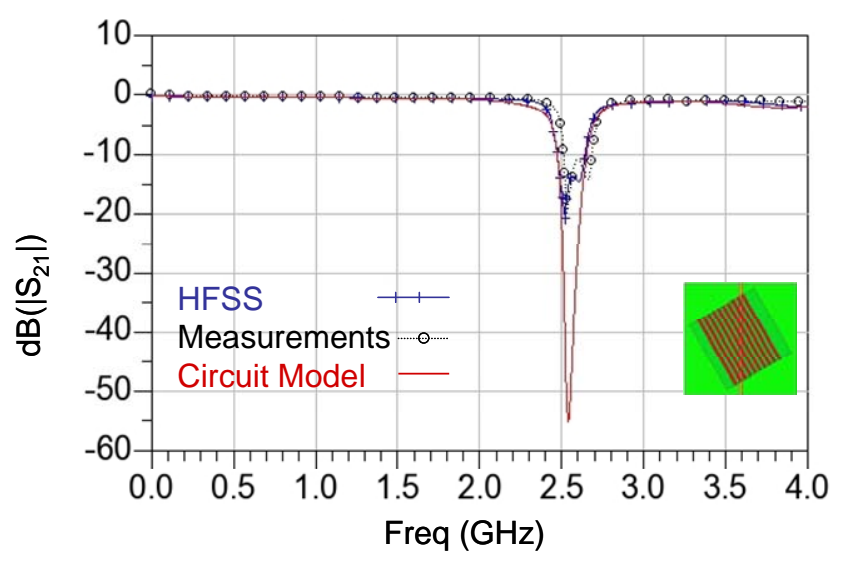

Fig 10-a : grid oriented at $30^{\circ}$

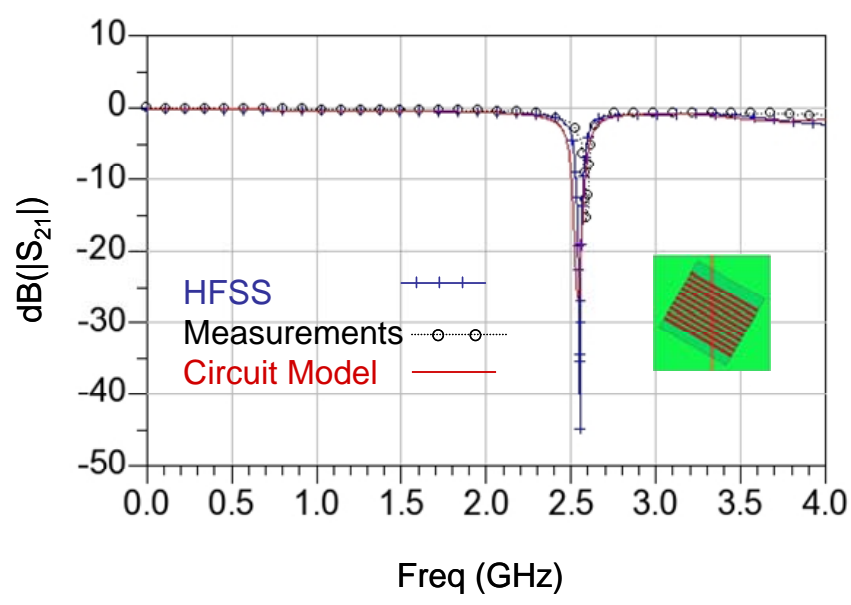

Fig 10-b : grid oriented at $60^{\circ}$

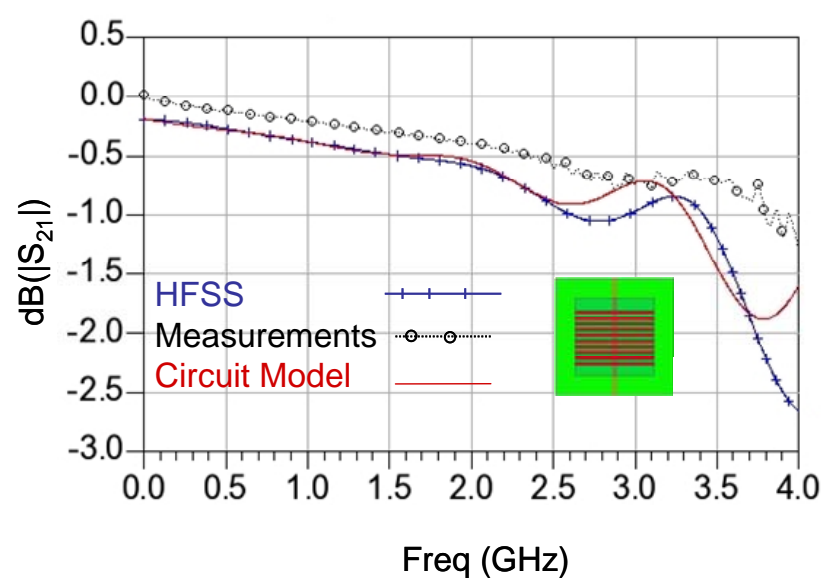

Fig 10-c : grid oriented at $90^{\circ}$

Fig. 10: S21 transmission parameter of a MTL in presence of floating interfering metal Grid.

In Fig. 10, we present the $S_{21}$ transmission parameters comparison in the $0-4 \mathrm{GHz}$ frequency band, between the measurements, the electromagnetic simulations and the circuit model results in the cases of a floating grid for $30^{\circ}, 60^{\circ}$ and $90^{\circ}$ orientations. We note the presence of a transmission zero around $2.5 \mathrm{GHz}$ for both cases $\theta=30^{\circ}$ and $\theta=60^{\circ}$, which can connect with the length of the grid as we showed it in the previous paragraph. All results concur, which validate first the independence of grid strip and also the circuit model which reflect the grid influence.

\section{Conclusion}

In this study, we presented the impact on the propagation characteristics of the orientation angle between an interfering metal strip and a microstrip transmission line. We showed the role of these parasitic lines on the frequencies at which transmission zeroes occur and we proposed a circuit model of these behaviours. We also studied the impact by metal grids on a transmission line in a multilayered PCB environment. We have proposed a simple circuit model which is coherent with measurements and EM simulations.

\section{References}

T. SUDO, and al "Electromagnetic Interference (EMI) of System-on-Package (SOP)" IEEE trans on Advanced Packaging Vol. 27, N. 2, May 2004 pp 304-314

Y. QUERE, and al «Frequency Domain Analysis of Transmission Zeroes on High-Speed Interconnects in the Presence of an Orthogonal Metal Grid Underlayer» IEEE trans on Advanced Packaging, Vol $31 n^{\circ} 4$, Nov 2008, pp: 684-691.

T. LE GOUGUEC, P.M. MARTIN “Impact by an Orthogonal Metal Grid upon Differential- and Common-Mode Characteristics of Coupled Lines in PCB Technology Structures" 14th IEEE workshop on Signal Propagation on Interconnects May 09-12 2010 Hildesheim Germany. 